\title{
Geomorfología, zonas de erosión y sedimentación costera de la Península de Burica, Costa Rica
}

\author{
Vanessa Rojas Herrera ${ }^{1,2,3}$ \\ 1. Fundación KETO, San José, Costa Rica. \\ 2. Escuela Centroamericana de Geología, Universidad de Costa Rica. \\ 3. Centro de Investigaciones en Ciencias Geológicas, Universidad de Costa Rica; vanroh@gmail.com
}

Recibido 11-VIII-2014. Corregido 20-XI-2014. Aceptado 22-XII-2014.

\begin{abstract}
Geomorphology, erosion and sedimentation in coastal zones of the Burica peninsula. The present work describes a geomorpholopic evaluation of the Burica peninsula, during 9 days in January 2008. Coastal geomorphology, erosion and depositional zones were evaluated between punta Banco and punta El Mangle. The most important morphologies found were structural origin morphologies, represented by active tectonic deformation, produced by the Panamá Fracture Zone, and the subduction of the Cocos ridge. The structural origin morphologies identified were, structural-denudational zones which dominate the study area, homocline in the south of the peninsula and structural terraces, that are observed easily between punta El Mangle and La Peña, and in punta Banco. Other morphologies were identified, such as marine, fluvial and denudational morphologies. The marine-origin morphologies include the beach, subdivided in subareas, separated due to the waves dynamic, the cliffs, that dominate the area between La Peña river and punta Banco, and the shore platforms in the area between punta El Mangle and La Peña. The denudational morphology is represented by landslides, which area present mainly in the cliffs. In the other hand, the fluvial origin morphologies, include La Peña, Peñita, Caña Blanca and Coco mouths and the floodplain of La Peña river. Intense erosional, slight to moderate erosional, sedimentational, and erosion and sedimentational zones were identified, having a great importance the coastal erosion, especially observed in the central and south of the peninsula. Considering the results and the interaction of them, the main hazards found are landslides, coastal erosion and the high seismicity of the area, which should be taken into account and studied in detail for a proper planning of the rural development. Rev. Biol. Trop. 63 (Suppl. 1): 407-416. Epub 2015 April 01.
\end{abstract}

Key words: Burica Peninsula, coastal geomorphology, natural hazards, structural morphology, denudational morphology, fluvial morphology.

La geología, los procesos geológicos y la geomorfología, tienen gran importancia en la biología, ya que pueden influir en las formas de vida y los tipos de ecosistemas (Bailey, 1985; Vartanyan, 2006; Bailey, 2009), inclusive, estos parámetros se utilizan cada vez más en los estudios de ecosistemas en sus diferentes escalas (Junta de Andalucía, 1997; Mardones, 2006). Esto puede observarse fácilmente, en zonas donde por la acidez de la roca, el estilo de la vegetación es diferente que en zonas con rocas de composición más básica, o en el caso de los procesos geológicos, estos van a determinar la geomorfología, y esta por ende la topografía y con ella los ecosistemas de un área específica (Bailey, 1985; Vartanyan, 2006).

La península de Burica, al sur de Costa Rica presenta morfologías variadas, desde terrazas estructurales hasta acantilados costeros, así como una rica historia geológica que se remonta desde el Cretácico ( 64Ma) hasta la actualidad, representada por rocas de origen volcánico y sedimentario (Corrigan, 1986; Obando, 1986; Di Marco, 1994; Buchs et al., 2009; Morell et al., 2011). El relieve del área, lleno de contrastes y riqueza escénica, 
es el resultado de todos estos procesos geológicos que han actuado en el área, pero sobre todo, la deformación tectónica del PleistocenoHoloceno (Fig. 1; Gardner et al., 1992; Morell et al., 2011). Dicha deformación ocurre debido a que esta península, se encuentra expuesta a esfuerzos tectónicos importantes, como por ejemplo la fractura de Panamá, una importante falla transformante tipo dextral, con rumbo norte-sur que se encuentra activa y que forma parte del límite entre la placa Coco y la placa Nazca (Morell et al., 2011). Esta interacción, unida a la colisión de la dorsal asísmica de Cocos (Corrigan, Mann \& Ingle, 1990; Gardner et al., 1992; Meschede \& Barckhausen, 2000), produce una gran complejidad tectónica en el área, que determina el paisaje y los procesos geológicos asociados.

En Costa Rica se han realizado varios estudios de geomorfología costera, con énfasis en los cambios geomorfológicos de un sector específico, entre los que destacan Lizano y Salas (2001), Cárdenes (2002), Cárdenes y Obando (2005) y Ortiz (2008). En estos trabajos se evalúan los cambios geomorfológicos costeros y /o sedimentológicos de diferentes regiones de la costa Pacífica, todos ellos, fuera del área de estudio. Sobresale por su importancia la evaluación realizada en la costa del Pacífico Central de Costa Rica por Cárdenes y Obando (2005), ya proponen y aplican por primera vez el método Índice de Erosión-Sedimentación Costera (IE-SC) con el cual aportan importantes herramientas para determinar la estabilidad costera y definir zonas con mayores problemas de erosión y/o sedimentación costera.

La presente investigación es un extracto de la evaluación del estado geológico-geomorfológico de la Península de Burica, con énfasis en este último, por lo cual se espera que sea un insumo para futuras investigaciones de detalle.

\section{MATERIALES Y MÉTODOS}

El sitio de estudio es la península de Burica, ubicada al suroeste de Costa Rica, entre las coordenadas geográficas $82.884092^{\circ} \mathrm{W}$ $08.000000^{\circ} \mathrm{N}$ a $83.154833^{\circ} \mathrm{W}-08.380288^{\circ}$

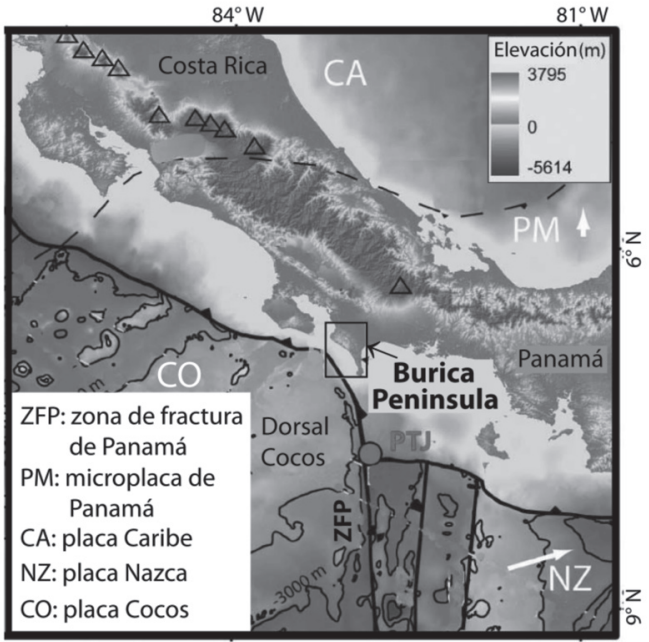

Fig. 1. Ubicación geográfica de la Zona de Fractura de Panamá (ZFP) y de otras estructuras tectónicas importantes. Fig. modificada de Morell et al. (2011).

Fig. 1. Localization of the Panamá Fracture Zone (ZPF) and others important tectonic structures. (After Morell et al., 2011).

N. Para acceder esta zona debe hacerse por vía marítima desde Punta Banco o por vía terrestre desde Panamá, se caracteriza por presentar un bosque muy húmedo montano bajo, con precipitaciones entre los 1850 a $4000 \mathrm{~mm}$ (Solano \& Villalobos, 2001). La península posee una topografía irregular de baja altitud, con elevaciones máximas de alrededor de $700 \mathrm{msnm}$, es angosta en el sector sur haciéndose más ancha conforme avanzamos hacia el norte.

Se recorrió la zona costera de la península durante 9 días de trabajo de campo durante el año 2008. En este periodo de tiempo se realizó un mapeo geológico y geomorfológico costero. Tomando en cuenta este último factor, se estudiaron y describieron tanto las macro morfologías, como por ejemplo las zonas denudacionales, acantilados costeros, etc., como las micro morfologías, en el caso las playas, en las cuales se separó y describió cada sector de la playa en función de la pendiente y granulometría predominante, así como se tomó en cuenta el ancho de estos sectores y el tipo y distribución de los fragmentos más grandes sedimentados (troncos, basura, etc.). Sin embargo, por 
efectos de escala, solo se presentan en el mapa las macro morfologías más importantes.

Se describieron las evidencias de los procesos erosivos y depositacionales costeros más relevantes (raíces expuestas, troncos caídos, terrazas de erosión, zonas de sedimentación actual, etc.), lográndose clasificar la zona en macroáreas donde actúan dichos procesos con diferente intensidad, y exponiéndose en este trabajo únicamente los resultados geomorfológicos y erosivos-depositacionales más sobresalientes. Además del cartografiado de campo, esta evaluación se complementó analizando la morfología del área mediante la observación de las hojas topográficas del IGN (1970), escala 1:50 000 y las fotografías aéreas CARTA 2005.

\section{RESULTADOS}

Geomorfología Costera: el área se caracteriza por presentar distintas morfologías originadas tanto por procesos estructurales, como denudacionales, fluviales y marinos, como se puede ver en las Figs. 2 y 3. En el trabajo Catálogo de Geomorfología Costera (Rojas, 2008a) pueden observarse fotografías de todas estas morfologías.

Morfologías de origen estructural: Estas dominan el área de estudio, y se pueden clasificar en zonas estructurales-denudacionales de baja a moderada pendiente, homoclinal y terrazas estructurales. Las primeras son las predominantes, tienen su origen gracias a la alta

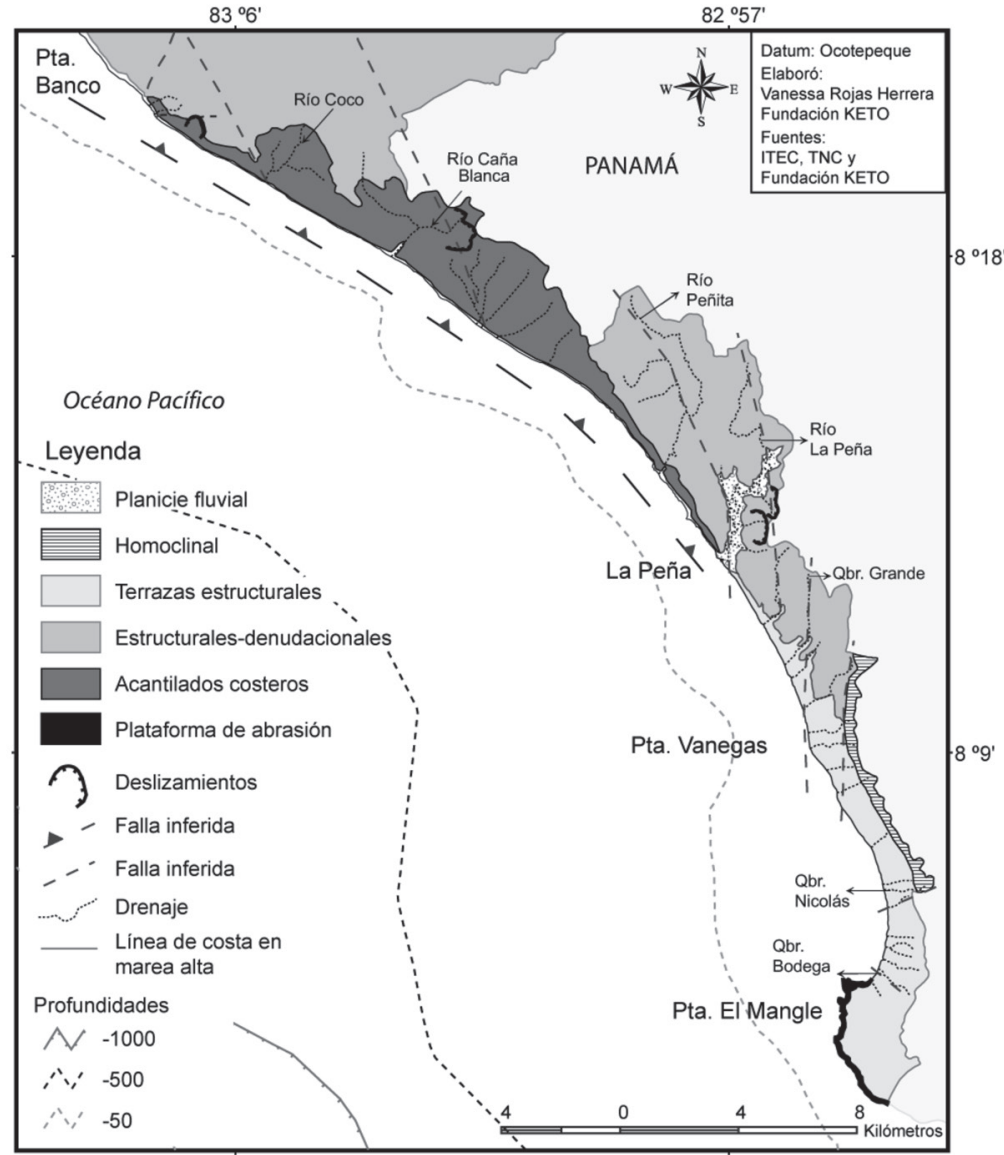

Fig. 2. Mapa geomorfológico del área. / Fig. 2. Geomorphologic map of the area. 


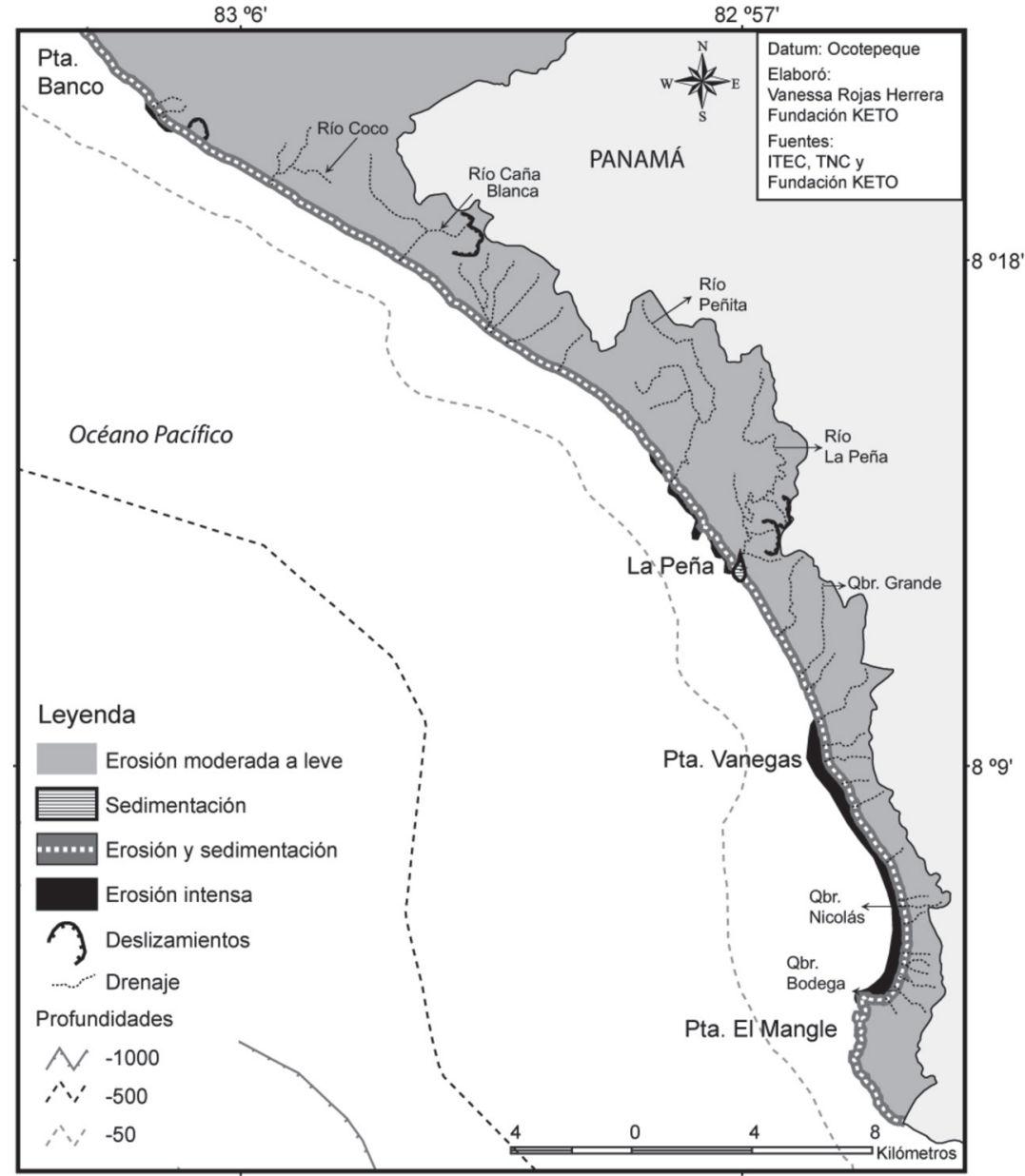

Fig. 3. Mapa de zonas de erosión y sedimentación. / Fig. 3. Erosion and sedimentation zones.

deformación tectónica Pleistocena-Holocena y procesos de denudación activos. Estas se ubican desde el norte de punta Vanegas hasta el noroeste del área, poseen una pendiente moderada a baja $\left(5^{\circ}\right.$ a $\left.15^{\circ}\right)$ y patrón de drenaje que varía desde subparalelo a paralelo, y subdendrítico.

Las terrazas estructurales son superficies semihorizontales que predominan en el sur de la península, presentan alturas métricas y un relieve horizontal hasta ligeramente inclinado. La disectación es leve y el patrón de drenaje es paralelo a subparalelo, estando constituidas por roca, suelo o ambos. Hacia el sector de punta El Mangle predominan las terrazas de más de $1.5 \mathrm{~m}$ de altura, generalmente constituidas por roca en la base y en la parte superior una superficie meteorizada. Hacia el sector de La Peña, las terrazas poseen una altura media de $1 \mathrm{~m}$ hasta ocasionalmente $1.8 \mathrm{~m}$ y están constituidas predominantemente por suelo. Mientras en Banco se observa una terraza compuesta por suelo, de $1 \mathrm{~m}$ aproximadamente, la cual está ligeramente disectada por algunas quebradas pequeñas.

El Homoclinal se ubica al sur de la península, sobresale el contraescarpe del mismo, cuyas capas de roca están inclinadas hacia el este, por lo que la mayor parte de esta morfología está fuera del área de estudio, hacia el este. En el área de estudio se observa una 
franja lineal con dirección norte-sur, con pendientes moderadas a fuertes con dirección oeste, moderada a leve disectación y patrón de drenaje paralelo. En fotografías aéreas puede observarse como las capas se inclinan hacia el este (Panamá), presentando flatirones, y un patrón de drenaje subparalelo.

Morfologías de origen denudacional: Se presentan a lo largo del área de estudio y constituyen las zonas donde están actuando los procesos de erosión. Las morfologías con este origen son los movimientos en masa o deslizamientos de suelo y/o roca; los cuales poseen tamaños variables (la mayoría no representables en el mapa de la Fig. 2, debido la escala). La altura máxima que pueden presentar es de $250 \mathrm{~m}$ en los acantilados costeros, ya que la pared inestable es más alta, mientras el ancho es variable, siendo el máximo de $100 \mathrm{~m}$ en algunas zonas de los acantilados y el área montañosa. Esta morfología predomina en los acantilados costeros debido a la fuerte pendiente, así como al grado de fracturamiento y meteorización de la roca expuesta, sin embargo, se reconocen también en las márgenes de los ríos más importantes y en el área montañosa. La susceptibilidad a deslizamientos de esta última puede deberse a la alta meteorización de la roca, dando como resultado espesores importantes de suelo y pendientes moderas.

Morfologías de origen fluvial: Se pueden dividir en planicie fluvial y zonas de desembocadura. La primera ha sido originada por el río La Peña en la parte central-sur de la península, posee $4 \mathrm{~km}$ de largo y $600 \mathrm{~m}$ de ancho en promedio, se caracteriza por poseer un pendiente muy baja $\left(<3^{\circ}\right)$, y estar constituida por gravas redondeadas a subredondeadas, centimétricas, de esfericidad media, dentro de una matriz de arena media a fina, generalmente cubiertos por arena media a fina. Las desembocaduras fueron separadas entre las formadas por quebradas y las formadas por ríos más grandes. Las primeras generalmente forman canales únicos en la parte alta de la playa mientras que, en la parte inferior presentan un patrón de drenaje dicotómico, y pequeños deltas en los que depositan sus sedimentos. Ocasionalmente, en la parte alta de la playa se observa el represamiento de las aguas debido a la gran cantidad de troncos, basura y otros restos de materia vegetal, que el oleaje deposita temporalmente.

Las desembocaduras más importantes, como la de Quebrada Grande y los ríos La Peña, Caña Blanca y Coco, muestran en su mayoría una barra arenosa de poca altura (entre $0,5 \mathrm{~m}$ a $1 \mathrm{~m}$ ) que desvía el cauce ligeramente paralelo a la playa y provocan represamientos pequeños (máximo $35 \mathrm{~m}$ por $20 \mathrm{~m}$ ).

Estas barras arenosas están constituidas por intercalaciones centimétricas de arenas finas a medias con arenas gruesas, compuestas por litoclastos $(70-90 \%)$, bioclastos $(30-10 \%)$, arena magnetítica $(<5 \%)$ y cuarzo $(<2 \%)$, cubiertas por troncos y basura. En el caso de la desembocadura del río La Peña, Caña Blanca y Coco, se observan entre uno a dos niveles de terrazas de alturas entre $0,5 \mathrm{~m}$ a $1,3 \mathrm{~m}$, horizontales a subhorizontales, constituidas por arenas finas a gruesas intercaladas con capas y lentes de grava dentro de una matriz de arena gruesa. Las arenas se componen de litoclastos (70-90\%), bioclástos $(30-10 \%)$, cuarzo $(<2 \%)$ y magnetita $(<2 \%)$, mientras las gravas por litoclastos $(100 \%)$. Cubriendo gran parte de las terrazas se observan fragmentos de ramas y troncos, basura como bolsas, botellas, tapas y en menor medida vidrio y otros.

Morfologías de origen marino: Son plataformas de abrasión, playas y acantilados costeros. Las plataformas de abrasión predominan en el centro-sur de la península y son especialmente importantes en el sector de punta El Mangle. Corresponden con zonas horizontales que son afectadas directamente por el oleaje, siendo cubiertas durante marea alta y quedando visibles durante marea baja. En esta área de punta El Mangle, la plataforma presenta un ancho de $500 \mathrm{~m}$ en promedio, desde la línea de marea alta hasta la línea de marea baja. Se observan mangles distribuidos de manera paralela a la costa y algunos parches aislados. Es importante recalcar que dicha plataforma presenta un intenso fallamiento con rumbo norte-sur. 
Hacia el sector central de la península (punta Venegas, La Peña) la plataforma se presenta como franjas discontinuas de menor ancho que al sur de la península (10m a $50 \mathrm{~m}$ durante el periodo entre mareas). Una de esas zonas corresponde con el incipiente tómbolo de La Peña (150m*100m), el cual se extiende paralelo a la costa y posee un relieve anguloso e irregular. Las franjas de plataforma del área de punta Banco tienen un ancho promedio de $50 \mathrm{~m}$ en el periodo entre mareas.

Las playas presentan diferencias marcadas especialmente en sus sistemas de sedimentación. Esto debido a que en el sur del área, las playas pueden subdividirse en zonas paralelas a la misma gracias a sus diferencias granulométricas y dinámica de sedimentación. Estas diferencias resultan en diferencias en la pendiente y en el ancho promedio de las mismas. En general, en el sur de la península, las playas se pueden dividir en tres zonas. La zona superior posee ancho variable, no superando los $10 \mathrm{~m}$, es discontinua, con pendientes de $5^{\circ}$ en promedio y en ella se observan gran cantidad de troncos de tamaños diversos, así como materia vegetal $\mathrm{y}$ todo tipo de basura. La zona intermedia tiene ancho variable que va desde los $3 \mathrm{~m}$ (punta El Mangle) hasta los 50m (punta Vanegas), posee pendientes de $2^{\circ}$ y esporádicamente hasta $10^{\circ}$. Constituida por arena fina a media de litoclastos $(70-90 \%)$, bioclastos $(30-10 \%)$ y magnetita $(<1 \%)$, y ocasionalmente arena gruesa en la parte inferior. Presenta estructuras sedimentarias como ripples, rills marks y crescent cast de tamaños centimétricos, así como bioturbación actual, que predomina en el sector de punta Vanegas. Por otro lado, poseen acumulaciones de grava de tamaños métricos, perpendiculares a la playa, aumentando su frecuencia hacia la desembocadura del río La Peña. La parte inferior de la playa se compone de arena fina con magnetita en la cual se forma una franja métrica de ondulitas, constituyendo el límite entre la zona arenosa y la plataforma de abrasión, sin embargo, hacia punta El Mangle, se produce una fuerte sedimentación temporal de madera y basura milimétrica a centimétrica en lugar de las ondulitas.
Habitualmente, desde la desembocadura del río La Peña hasta punta Banco, las playas son más angostas y solamente se distinguen dos zonas, la superior e inferior. La primera posee anchos métricos $(<10 \mathrm{~m})$, pendientes de $10^{\circ}$ y la ocurrencia de acumulaciones y franjas métricas de grava y bloques, mientras que la depositación de madera y basura disminuye considerablemente hasta hacerse nula en algunos sectores, con excepción de punta Banco. La grava y los bloques presentan mayor tamaño y densidad hacia las salientes costeras y al pie del acantilado. La zona inferior tiene un ancho promedio de $15 \mathrm{~m}$ y ocasionalmente de $70 \mathrm{~m}$, pendiente de $5^{\circ}$ a $10^{\circ}$ y el substrato se compone de arena media a gruesa compuesta por litoclastos $(70-90 \%)$, bioclastos (30-10\%), cuarzo $(<2 \%)$, radiolarita $(<2 \%)$ y magnetita $(<2 \%)$. Presentan estructuras sedimentarias como crescent cast, ripples, ondulitas, y estructuras de bioturbación actual, etc., sobre todo en punta Banco. Esporádicamente, en la parte baja de la zona arenosa se presenta arena gruesa y grava fina, constituyendo el límite entre esta y la plataforma de abrasión.

Se reconocen acantilados costeros principalmente al norte del área desde el sector de La Peña hasta punta Banco. Como parte de estos, se ha incluido todo el escarpe montañoso de alta pendiente $\left(15^{\circ}\right.$ a $\left.30^{\circ}\right)$ que se encuentra en dicho sector. Estos acantilados presentan una pendiente mayor a $15^{\circ}$ y pueden alcanzar en algunos sectores $\approx 90^{\circ}$, están de parcial a totalmente cubiertos de vegetación, de alta a moderadamente disectados y presentan un patrón de drenaje paralelo a subparalelo. Un gran porcentaje del frente del acantilado (sobre todo en la parte baja) presenta deslizamientos. La diferencia entre los acantilados ubicados al sur de la quebrada La Peñita con los ubicados al norte, es que la altura de los primeros es menor en promedio.

Zonas de erosión y sedimentación: Se distinguieron cuatro diferentes de zonas a gran escala, las zonas de erosión intensa, las de erosión moderada a leve, las zonas de sedimentación y las zonas en donde ocurre tanto 
erosión como sedimentación (Fig. 3; para más detalle ver Catálogo de zonas de erosión y depositación en Rojas, 2008b). Las zonas de erosión intensa corresponden con la plataforma de abrasión marina, las salientes rocosas costeras, el frente costero de terrazas estructurales y los acantilados marinos. Todos estos sectores están propensos a gran erosión por parte del oleaje, así como por deslizamientos en el caso de los acantilados costeros. Con respecto al frente costero de las terrazas, este sufre erosión intensa, ya que el oleaje va socavando su base y dejando al descubierto raíces de palmeras y árboles, se observaron raíces expuestas, palmeras y árboles inclinados hacia la playa y algunos totalmente caídos sobre la misma.

Las zonas de erosión moderada a leve constituyen las áreas montañosas sujetas a procesos erosivos de denudación y disectación. La erosión en esas zonas se produce por la escorrentía superficial provocada por la lluvia, el lavado de los ríos sobre el suelo y la roca, así como por los deslizamientos presentes en esta área. Las zonas de erosión y sedimentación son las playas, las cuales pueden dividirse en microzonas paralelas a la costa debido a la dinámica de estos procesos. En la parte baja de la playa se produce sedimentación moderada a baja, en la parte intermedia de la playa se produce tanto erosión como sedimentación leve, mientras la parte superior se caracteriza por una depositación temporal de troncos y basura. Las zonas de sedimentación comprenden las desembocaduras de las quebradas y ríos más importantes del área, como río La Peña, Peñita, Caña Blanca y Coco, en las cuales puede observarse una estrecha zona de barras de sedimentación de material de espesores métricos, producto de la combinación de la acción marina y fluvial.

\section{DISCUSIÓN}

Se reconoce la predominancia de las morfologías de origen estructural: zonas estructurales-denudacionales de baja a moderada pendiente, homoclinal y terrazas estructurales, y en menor medida las de origen denudacional: los deslizamientos, de origen fluvial: planicie aluvial y desembocaduras y de origen marino: playas, plataformas de abrasión y acantilados costeros, concordando con las descripciones, desde el punto de vista de morfologías estructurales, de Obando (1986) y Morell et al. (2011).

Para una adecuada planificación territorial es necesario tomar en cuenta muchos aspectos, como lo son la litología, la condición geodinámica, la condición hidrogeológica, estabilidad de ladera y susceptibilidad a las amenazas naturales, esto de acuerdo con los lineamientos establecidos mediante la resolución $\mathrm{N}^{\circ}$ 588-97 de SETENA, publicada en La Gaceta en 1997 para el Ordenamiento Ambiental del Territorio (OAT), sin embargo, en el presente estudio únicamente se realizó una evaluación geológica rápida de la costa, por lo tanto, tomando en cuenta las limitantes de este estudio, solo se discutirán a continuación los aspectos considerados como los más sobresalientes del área, siendo estos los deslizamientos, la erosión costera y la alta sismicidad.

Geomorfológicamente, se constató que el área está fuertemente sujeta a procesos de deformación tectónica, ya que es posible observar una predominancia de morfologías de origen estructural, entre ellas diversas trazas de fallas (Fig. 2; fallas observadas tanto en campo como en fotografía aérea).

De acuerdo con las evidencias observadas en el área, tales como, diferentes alturas entre los frentes costeros de las terrazas, así como diversos grados de meteorización (porcentaje de suelo) en distintos sectores del área, se puede interpretar que toda la zona ha sido afectada en diferentes proporciones por el levantamiento costero que formó las terrazas actuales. Dichas terrazas fueron detalladamente estudiadas por Morell et al. (2011), quienes describen y determinan la existencia de al menos 8 niveles de terrazas en la península, tanto del lado costarricense como panameño. Con las dataciones realizadas, estos autores obtienen tazas de levantamiento costero que van desde $2.1 \pm 0.1$ y $7.7 \pm 0.5 \mathrm{~mm} /$ año para las terrazas más antiguas y $6.9 \pm 1.0$ y $19.3 \pm 8.0 \mathrm{~mm} /$ año para la terrazas del Holoceno Tardío, concluyéndose que la 
taza de levantamiento tectónico de la península ha aumentado en periodos más recientes y que las mismas son contemporáneas a los eventos sísmicos del área. De ahí la importancia de este factor en el diseño constructivo y planificación rural a futuro. Es importante resaltar que tan solo en los últimos 100 años, han ocurrido tres sismos de magnitud mayor a 7, en 1934 (magnitud 7.6), 1941 (magnitud 7.3) y 1983 (magnitud 7.3) (Camacho, 1993; RSN, s.f.a; s.f.b; s.f.c). En años más recientes ocurrieron en 2002, 2003 y 2004 sismos mayores a $6.6 \mathrm{M}_{\mathrm{w}}$ (RSN, s.f.d; s.f.e; s.f.f).

Desde el punto de vista de zonas de erosión y sedimentación, se observaron zonas de erosión intensa, zonas de erosión moderada a leve, zonas de sedimentación y zonas en donde ocurre tanto erosión como sedimentación. Las zonas más importantes a tomar en cuenta serían los deslizamientos y la erosión costera. Los deslizamientos se encuentran sobre todo en la parte norte de la península y son fácilmente apreciables en los acantilados costeros y en las márgenes del río La Peñita. Sin embargo, también son frecuentes en las áreas montañosas en donde se ubican los caminos y algunas casas de la población Guaymí. Las evidencias de esta inestabilidad de laderas son troncos de árboles inclinados hacia la pendiente de la ladera, raíces descubiertas, grietas en el suelo y desprendimiento de la capa superficial del suelo. Es importante destacar que los deslizamientos pueden migrar hacia atrás (montaña arriba) y/o hacia los lados. Lo anterior acarrea cada vez más material, el cual, dependiendo del volumen de roca removido, puede causar represamientos en los cauces de los ríos, con su posterior desborde e inundaciones, matando gran cantidad de peces y organismos de agua dulce, así como marinos (Cortés, Soto \& Jiménez, 1994).

Considerando la inestabilidad de laderas existente en los sectores de alta a moderada pendiente y al ser esta una zona de alta sismicidad, se debe tomar en cuenta que este último factor sería un efecto disparador de nuevos deslizamiento o reactivador de deslizamientos antiguos, por lo cual se recomienda que estas áreas sean utilizadas para conservación del bosque, ya que el establecimiento de nuevas actividades humanas en la zona podrían aumentar el riesgo de afectación.

En el sector sur de la península las terrazas estructurales están fuertemente erosionadas, es posible observar gran cantidad de raíces de palmeras y árboles expuestas, así como estas mismas, dobladas o caídas hacia la playa, además, gran cantidad de restos de palmeras y árboles en la parte superior de la playa que han sido erosionados anteriormente. Este fenómeno erosivo podría tener su origen en la dinámica del oleaje costero, por ejemplo, un aumento en la energía del oleaje, entre otros (Bird, 2008), así como podría representar un ligero hundimiento tectónico asociado a un periodo intersísmico, sin embargo, en este trabajo no se pudo comprobar ninguna de estas hipótesis, por lo que se recomienda hacer estudios específicos al respecto.

Tomando en consideración las narraciones de los habitantes de la zona consultados en el 2008, ellos expresaron que la erosión puede alcanzar una velocidad ligeramente menor a medio metro por año aproximadamente, por lo que esto podría representa un problema potencial que debe ser estudiado en detalle y mediante diversos métodos para analizar su evolución a corto, mediano y largo plazo. Se recomienda aplicar la metodología propuesta por Cárdenes y Obando (2005), la cual pretende analizar los procesos de erosión-sedimentación costera mediante métodos semi-cuantitativos, durante un rango de tiempo no menor de dos años. Aparte de esto, realizar una reconstrucción de la evolución costera por medio de fotografías aéreas e imágenes de satélite de diferentes años, todo esto unido a una evaluación de la percepción de la población a lo largo de un periodo de tiempo establecido. Por último, considerando estos factores erosivos se recomienda respetar las regulaciones de la Zona Marítimo Terrestre.

\section{AGRADECIMIENTOS}

Este trabajo no hubiera sido posible sin el financiamiento de The Nature Conservancy 
(TNC) y Fundación Keto y sin la colaboración de la geóloga Diana Gamboa Montoya y la compañía y guía a lo largo del área de Nicolás Santos. Se agradece al Proyecto Consolidación de Áreas Marinas Protegidas del Sistema Nacional de Áreas de Conservación, Programa de Naciones Unidas para el Desarrollo y el Global Environment Facility (GEF) por su colaboración en la publicación.

\section{RESUMEN}

Se efectuó una evaluación geomorfológica y de zonas de erosión y sedimentación del sector oeste de la Península, desde punta Banco hasta punta El Mangle, con el fin de determinar aspectos relevantes desde el punto de vista geológico. El estudio se llevo a cabo durante 9 días en enero del 2008, tiempo en el cual, se realizó el estudio geológico, geomorfológico y de zonas de erosión-sedimentación costera, presentándose en este trabajo solamente los resultados relevantes de los últimos dos aspectos. Geomorfológicamente, se reconocen una predominancia de morfologías de origen estructural, y en menor grado de origen marino, fluvial y denudacional. Se definieron cuatro zonas de erosión-sedimentación, zonas de erosión intensa, de erosión moderada a leve, de erosión y sedimentación y zonas de depositación. Los procesos geológicos más importantes son los deslizamientos, la erosión costera y la alta sismicidad del área, factores que deben ser tomados en cuenta para una adecuada planificación rural de la península

Palabras clave: Península de Burica, geomorfología costera, amenazas naturales, morfologías estructurales, morfologías denudacionales, morfologías fluviales.

\section{REFERENCIAS}

Bailey, R. (1985). The factor of scale in ecosystem mapping. Environmental Management, 9(4), 271-276.

Bailey, R. (2009). Ecosystem Geography, from ecoregions to sites. New York: Springer.

Bird, E. (2008). Coastal geomorphology, and introduction. England: Jhon Wiley \& Sons.

Buchs, D., Baumgartner, P., Baumgartner-Mora, C., Bandini, A., Jackett, J., Diserens, O., \& Stucki, J. (2009). Late Cretaceous to Miocene seamount accretion and mélange formation in the Osa and Burica peninsulas (southern Costa Rica): Episodic growth of a convergent margin. Geological Society Special Publication, 328, 411-456.

Camacho, E. (1993). La amenaza de terremotos en el Istmo de Panamá. Revista Universidad, 49, 285-303.
Cárdenes, G., (2002). Sedimentología de los ambientes costeros y continentales actuales y sus implicaciones geológico-ambientales en la región de Parrita, Pacifico Central de Costa Rica (Tesis de Licenciatura), Escuela Centroamericana de Geología, Universidad de Costa Rica, Costa Rica.

Cárdenes, G., \& Obando, L., (2005). Índice de erosiónsedimetación costera (IE-SC): Una aplicación en la costa del Pacífico Central de Costa Rica. Revista Geológica de América Central, 32, 33-43.

Corrigan, J. (1986). Geology of the Burica Peninsula, Panamá-Costa Rica: Neotectonics implications for the southern middle America convergent margin (Doctoral dissertation). University of Texas, USA.

Corrigan, J., Mann, P., \& Ingle, J. (1990). Forearc response to subduction of the Cocos Ridge, Panama-Costa Rica. Geological Society of America Bulletin, 102, 628-652.

Cortés, J., Soto, R., \& Jiménez, C., (1994). Efectos ecológicos del terremoto de Limón. Revista Geológica de América Central, 187, 192.

Di Marco, G. (1994). Les terrains accrétés du sud du Costa Rica: Evolution tectonostratigraphique de la marge occidentale de la plaque Caraibe (Doctoral dissertation). Université de Lausanne, Switzerland.

Gardner, T., Verdnock, D., Pinter, N., Slingerland, R., Furlong, K., Bullard, T., \& Wells, S. (1992). Quaternary uplift astride the aseismic Cocos Ridge, Pacific coast, Costa Rica. Geological Society of America Bulletin, 104, 219-232.

IGN. (1970). Hoja Topográfica Burica. Escala 1:50.000. Instituto Geográfico de Costa Rica.

IGN. (1970). Hoja Topográfica Pavón. Escala 1:50.000. Instituto Geográfico de Costa Rica.

IGN. (1970). Hoja Topográfica Río Caña Blanca. Escala 1:50.000. Instituto Geográfico de Costa Rica.

IGN. (1970). Hoja Topográfica Puerto Armuelles. Escala 1:50.000. Instituto Geográfico de Costa Rica.

Junta de Andalucía. (1997). Reconocimiento biofisico de espacios naturales protegidos: Doñana, una aproximación ecosistémica. España: Consejería del Medio Ambiente: Andalucía.

Lizano, O., \& Salas, D. (2001). Variaciones geomorfológicas en los últimos 50 años de la isla Damas, Quepos, Costa Rica. Revista de Biología Tropical, 49(2), 171-177.

Mardones, G. (2006). Clasificación jerárquica y cartografía de ecosistemas en la zona andina de la región de Biobío, Chile. Revista de Geografia Norte Grande, $35,59-75$.

Meschede, M., \& Barckhausen, U. (2000). Plate tectonic evolution of the Cocos-Nazca spreading center. In E. Silver, G. Kimura \& T. H. Shipley (Eds.), 
Prodcedings ODP, Science Results (pp. 1-10). USA: College Station.

Morell, K., Fisher, D., Gardner, T., La Femina, P., Davidson, D., \& Teletzke, A. (2011). Quaternary outer fore-arc deformation and uplift inboard of the Panama Triple Junctions, Burica Peninsula. Journal of Geophysical Research, 116, B05402.

Obando, J. (1986). Sedimentología y tectónica del Cretácico y Paleógeno de la región de Golfito, Península de Burica y Península de Osa, Provincia de Puntarenas, Costa Rica (Tesis de Licenciatura). Escuela Centroamericana de Geología, Universidad de Costa Rica, Costa Rica.

Ortiz, E. (2008). Nota Técnica: Cambios geomorfológicos en el litoral Caribe y Pacífico de Costa Rica. Caso del Complejo Deltáico Sierpe. Kurú, Revista Forestal Mesoamericana, 5(15).

Red Sismológica Nacional. (1934) (s. f. a): Terremoto de Puerto Armuelles, reporte julio 1934. (Informe interno. 2 p.). San José, Costa Rica: Red Sismológica Nacional.

Red Sismológica Nacional. (1941) (s. f. b): Terremoto de Osa, reporte diciembre 1941. (Informe interno. 2 p.) San José, Costa Rica: Red Sismólogica Nacional.

Red Sismológica Nacional. (1983) (s. f. c): Terremoto de Golfito o del Sábado Santo, reporte abril 1983.
(Informe interno. 2 p.). San José, Costa Rica: Red Sismólogica Nacional.

Red Sismológica Nacional. (2002) (s. f. d): Terremoto de Burica, reporte julio 2002. (Informe interno. 3 p.). San José, Costa Rica: Red Sismólogica Nacional.

Red Sismológica Nacional. (2003) (s. f. e): Terremoto de Puerto Armuelles, reporte diciembre 2003. (Informe interno. 3 p.). San José, Costa Rica: Red Sismólogica Nacional.

Red Sismológica Nacional. (2004) (s. f. f): Sismo 4 de febrero del 2004, reporte febrero 2004. (Informe interno, 2 p.). San José, Costa Rica: Red Sismólogica Nacional.

Rojas, V. (2008a). Catálogo de Geomorfología Costera. Proyecto Marino Conte Burica, Puntarenas Costa Rica. Costa Rica: Fundación Keto.

Rojas, V. (2008b). Catálogo de Zonas de Erosión y sedimentación. Proyecto Marino Conte Burica, Puntarenas Costa Rica. Costa Rica: Fundación Keto.

Solano, J., \& Villalobos, R. (2001). Aspectos fisiográficos aplicados a un bosquejo de regionalización geográfico climático de Costa Rica. Tópicos Meteorológicos y Oceanigráficos, 8(1), 26-39.

Vartanyan, G. (2006). Influence of modern geological processes on evolution of ecosystems. In I. Zektser (Ed.), Geology and Ecosystems (pp. 15-20). USA: Springer. 